\title{
Apprendre à lire, à écrire et à parler de sciences : un modèle pour guider la croissance d'une communauté d'apprentissage didactique
}

\author{
Liliane Dionne \\ Université d'Ottawa
}

\begin{abstract}
Résumé
La communauté d'apprentissage « didactique » visant spécifiquement le développement professionnel des enseignants en sciences nécessite que des études plus approfondies soient effectuées pour mieux accompagner les praticiens dans l'ajustement de leur répertoire de pratiques en enseignement des sciences et de la technologie. Une analyse critique du concept de littératies multiples fournit certaines assises pour orienter ces communautés d'apprentissage didactiques en sciences et en technologie, en fournissant des modèles et des outils pour guider l'accompagnement. Étant orienté spécifiquement vers les sciences, le modèle «d'Investigation guidée pour le développement des littératies multiples » (IGdLM) est susceptible d'inspirer les enseignants (Palincsar, Magnusson, Marano, Ford et Brown, 1998) au sein d'une communauté d'apprentissage didactique. Travailler conjointement à développer des pratiques pédagogiques qui incitent les élèves à lire, écrire et parler de sciences peut guider l'enseignant pour améliorer l'apprentissage des sciences en classe (Lemke, 1990). Ce texte aborde également le contexte minoritaire francophone en Ontario et le double défi qu'il présente pour les enseignants qui désirent ajuster leur répertoire de pratiques dans le domaine des sciences et de la technologie.
\end{abstract}

\section{Abstract}

Learning community, more specifically "didactic" learning community directed toward professional development of teachers in science teaching, needs specific attention from the research community. In this endeavor, and from the perspective of a critical analysis of the literature in this area, the concept of multiple literacy and, more specific to science teaching and learning, the model of "Guided inquiry supporting multiple literacies" (GISML) could provide teachers with many insights in their efforts to adjust their practices in science teaching (Palincsar Magnusson, Marano, Ford, and Brown, 1998) in a didactic learning community. Working collectively to help students to better read, write and speak science could help teachers to better understand science teaching (Lemke, 1990). This article also discusses the double challenge faced by Ontario teachers working in elementary schools in French minority settings, and who want to adjust their teaching practices in science and technology. 


\section{La problématique liée à l'ajustement des pratiques pédagogiques en sciences et technologie}

L'ajustement des pratiques pédagogiques consiste dans le travail des enseignants qui veulent améliorer leur enseignement et élargir leur répertoire de pratiques pour améliorer l'apprentissage chez leurs élèves. II peut signifier également la transformation des pratiques pédagogiques en vue de les adapter à une réforme curriculaire (SavoieZajc et Descamps-Bednarz, 2007). Malgré leurs nombreuses tentatives pour ajuster leurs pratiques pédagogiques en sciences et en technologie, il demeure que, pour de nombreux praticiens, passer de l'enseignement traditionnel vers des modes socioconstructivistes ressemble à un voyage sur une mer tumultueuse (Wasley, Donmoyer et Maxwell, 1995). En examinant la nature des activités en sciences et en technologie proposées en salle de classe, il s'avère que l'approche la plus courante se traduit par la « leçon explicative » (Couture, 2005). Ces explications et illustrations s'éloignent pourtant d'un enseignement exemplaire en sciences qui consiste essentiellement à faire émerger des questions chez les élèves et à soutenir ceux-ci dans leur démarche de construction des connaissances scientifiques (Tobin, Tippins et Gallard, 1994). L'apprentissage des élèves se trouve stimulé lorsque les pratiques pédagogiques s'orientent vers le développement d'habiletés scientifiques et sociales, et la construction de sens en sciences. Une conception empiriste de l'enseignement basée sur l'observation et l'expérimentation traditionnelle néglige les processus de conceptualisation ou de métacognition nécessaires pour la construction des connaissances scientifiques chez l'élève (Couture, 2005).

Plusieurs mécanismes concrets sont requis pour accompagner les enseignants dans l'enrichissement de leur répertoire de pratiques pédagogiques en sciences et pour passer d'une approche traditionnelle à une approche constructiviste (Larochelle et Bednarz, 1994; Morais, Neves et Afonso, 2005). Comment amener les éducateurs à transformer des modes d'enseignement axés sur la mémorisation en modes axés sur l'application des connaissances en contexte? Comment amener les enseignants à passer de la présentation de contenus spécifiques, à une démarche de résolution de problèmes et à remplacer l'approche mono-disciplinaire par l'interdisciplinarité (Magnusson et Palincsar, 1995)? Pour aider les élèves à mieux appréhender les savoirs scientifiques, une des approches à être privilégiées par l'enseignant consiste à mieux intégrer la démarche d'investigation aux pratiques pédagogiques en sciences.

Le développement professionnel est souvent considéré comme un élément clé dans la mise en œuvre des réformes en éducation scientifique (Rhoton et Stiles, 2002). Idéalement, le développement professionnel doit s'échelonner du début de la formation initiale de l'enseignant jusqu'à la fin de sa carrière. Ce perfectionnement vise à 
intégrer à la pratique enseignante les innovations pédagogiques au service de l'apprentissage de l'élève. II a pour objectif de permettre au praticien de choisir et de s'approprier les pratiques pédagogiques les plus favorables, en fonction de la nature des apprentissages ciblés et des besoins des élèves.

Ces dernières années, les études sur le développement professionnel des enseignants ont mis l'accent sur le potentiel que revêtent certains dispositifs collectifs et, en particulier, ceux imbriqués directement à la tâche (Cochran-Smith et Lytle, 1999; Seidel Horn, 2003; Sparks, 1999). Les communautés d'apprentissage, auxquelles s'ajoutent les groupes de recherche et les communautés de pratique, sont de plus en plus reconnues comme des dispositifs susceptibles de soutenir l'enseignant vers un changement durable de ses pratiques pédagogiques (Cochran-Smith et Lytle, 1999). Les recherches en cours actuellement dans le domaine du développement professionnel (DP) révèlent que l'apprenant adulte profite de la synergie engendrée par les échanges et les dialogues entre collègues qui se produisent dans ces dispositifs de DP de type socioconstructiviste. De plus, les enseignants apprennent davantage lorsqu'ils peuvent choisir leur projet en lien avec leur pratique, tout en étant libre d'adhérer au groupe (Dionne, 2003). Trois conditions apparaissent essentielles pour assurer le succès d'une formation continue de type constructiviste : 1) l'objet d'apprentissage doit faire partie des préoccupations des acteurs en devenant « leur projet », selon un principe d'appropriation ; 2) le dispositif de formation doit fournir un lieu d'échange ou de dialogue à l'horizontal, entre pairs, et avec une personne-ressource qui se distancie de la posture d'expert; 3) la structure du dispositif doit assurer une dimension réflexive aux dialogues, par le processus métacognitif qui se met en place et qui donne aux praticiens le pouvoir de créer de nouvelles idées et, ultimement, d'ajuster leurs pratiques pédagogiques (Savoie-Zajc et Dionne, 2006).

Pour favoriser l'enrichissement du répertoire de pratiques en sciences et en technologie, la communauté d'apprentissage (CA) apparaît pertinente de façon globale. La CA peut être vue comme un groupe d'individus qui se rassemblent dans le but d'apprendre, de se soutenir les uns les autres et de développer une vision commune dans l'école (Schussler, 2003). Par contre, peu d'études nous renseignent sur la CA didactique, soit celle qui vise à accompagner les enseignants pour ajuster leur répertoire de pratiques dans un domaine spécifique comme l'enseignement des sciences et de la technologie.

À l'ordre d'enseignement élémentaire, des modèles pertinents de CA sont nécessaires pour guider le développement professionnel en sciences et en technologie. II s'avère important d'offrir un cadre aux enseignants pour orienter leur travail conjoint, tel le modèle des littératies multiples, associé à des modèles spécifiques aux sciences, comme le modèle d'investigation guidée pour le développement des littératies multiples (IGdLM) (Palincsar, Collins, Marano et Magnusson, 2000). Ce dernier modèle propose à l'enseignant des stratégies pour accroître l'apprentissage des élèves dans le domaine des sciences. Mais avant de présenter ce modèle plus en détail, il convient d'examiner le contexte spécifique de l'apprentissage des sciences dans les écoles francophones en Ontario, et donner un aperçu de l'avancement de certains travaux dans le domaine du développement professionnel des enseignants. 
Les particularités du milieu minoritaire francophone : un double défi pour l'enseignement des sciences et de la technologie

L'enseignement et l'apprentissage des sciences dans une école francophone en milieu minoritaire comportent un double défi. Ils renvoient au défi évoqué précédemment, celui de la résistance des éducateurs pour supplanter un enseignement traditionnel. Mais ils réfèrent aussi au défi de la maitrise de la langue française, qui est souvent une langue étrangère pour l'élève qui fréquente cette école. Malgré les mesures adoptées par le ministère de l'Éducation de l'Ontario pour contrer ce phénomène, les taux d'échec scolaire en sciences demeurent élevés dans les milieux francophones minoritaires (CMEC, 2004). D'après Herry (2000), seulement 45\% des élèves interrogés parlent toujours français à la maison et cette réalité revêt une importance particulière pour l'enseignement des sciences. Ce lien entre l'utilisation de la langue et le rendement en sciences peut vouloir dire que l'apprentissage des sciences est fortement lié à la maîtrise de la langue, dans la mesure où celle-ci sert de véhicule aux concepts et aux processus de résolution de problèmes inhérents aux sciences. Dans ce contexte, les littératies multiples offrent à l'enseignant un cadre de renforcement des habiletés langagières, tout en donnant une plus large orientation à l'apprentissage des sciences.

Les attentes sont nombreuses envers le personnel enseignant qui évolue dans une école minoritaire francophone. On demande aux praticiens d'intervenir en tant qu'agents de transmission culturelle aussi bien que de démontrer un haut niveau de compétence dans leurs responsabilités éducatives. Pour y parvenir, ils doivent disposer de tous les outils leur permettant de saisir les enjeux de l'enseignement en milieu minoritaire. En plus de vivre dans un contexte éducationnel en perpétuel changement, où les connaissances et les compétences doivent être sans cesse remises à jour et perfectionnées, la tâche des enseignants est encore plus complexe lorsqu'ils évoluent dans les milieux où l'anglais est prédominant et où l'utilisation du français à la maison est faible (FCE, 2004). En effet, les praticiens doivent pouvoir mettre en place une pédagogie qui tient compte de ce problème de langue, et utiliser des stratégies pour contrer l'insécurité linguistique (Bryan, Reveles et Kelly, 2005). Pour encourager les élèves à parler et à communiquer leurs conceptions scientifiques, il faut privilégier une attitude ouverte à la variation linguistique. Cette attitude consiste à ouvrir le cadre d'apprentissage en acceptant le langage vernaculaire des élèves, dans le but de provoquer des changements dans les représentations linguistiques (Cormier, Pruneau, Rivard et Blain, 2004).

Au sein des écoles francophones en Ontario, on assiste présentement à la montée d'un dispositif qui sert principalement à mettre en place les changements et améliorations pédagogiques apportés par le ministère de l'Éducation et les conseils scolaires. II s'agit des communautés d'apprentissage professionnelles ou CAP (Dufour et Eaker, 2004). Les CAP se proposent comme un lieu où les enseignants peuvent échanger au niveau de leurs stratégies afin de mieux aider les élèves les plus faibles à atteindre les standards de réussite attendus (AEFO, 2006). L'objectif spécifique de ces CAP dans les écoles françaises de l'Ontario se concentre sur l'apprentissage de la littératie et de la numératie. À l'intérieur de ces communautés, la mission consiste à améliorer l'apprentissage du français, à promouvoir la langue française, et à développer les compétences en mathématiques chez les élèves. La proposition qui est faite dans ce texte concerne l'émergence d'un autre type de communauté d'apprentissage ou CA qui se destine plutôt à accompagner les enseignants dans l'ajustement de leurs pratiques pédagogiques en sciences et en 
technologie. En proposant aux enseignants des modèles spécifiques pour guider leur DP en sciences, les praticiens pourraient entrevoir l'enseignement des sciences et de la technologie comme un moyen de renforcement de la littératie et de la numératie. En étant différente et complémentaire aux CAP, la CA apparaît tout à fait compatible avec les orientations des CAP actuelles. Cette CA cible un curriculum en particulier, dans le cas qui nous intéresse ici, celui des sciences et de la technologie. Elle s'oriente spécifiquement vers le DP des enseignants en sciences et en technologie et a comme retombée l'enrichissement de la culture scientifique des élèves. Pour développer les littératies multiples en milieu minoritaire francophone, et en particulier la littératie scientifique, la CA se propose comme un dispositif susceptible de répondre aux besoins des enseignants en complémentarité aux CAP, tout en lui permettant de satisfaire au développement de la littératie langagière et de la numératie chez les élèves.

\section{Aperçu de la recherche sur le développement professionnel des enseignants, en général et en sciences}

Le développement professionnel (DP) se définit comme toute forme d'apprentissage professionnel dans lequel les enseignants s'engagent librement, trouvent des solutions aux problèmes qu'ils rencontrent, développent une nouvelle compréhension et ajustent leurs pratiques pédagogiques en conséquence (Wells, 1993). Certains auteurs affirment que le DP devrait être plus global au lieu de viser seulement la transformation des pratiques pédagogiques chez les praticiens. Cette transformation devrait être accompagnée d'une meilleure compréhension des fondements didactiques en sciences et de la nature de la science (Abd-El-Khalick et Lederman, 2000). Selon Fullan, Bennett et Rolheiser-Bennett (1990) le DP comporte quatre dimensions qui englobent les pratiques pédagogiques, mais aussi la vision de soi, les habiletés de recherche et les habiletés de collaboration. Un processus de DP complet devrait rejoindre toutes ces dimensions et ne pas se concentrer uniquement sur les pratiques pédagogiques. Le travail réflexif engendré par des dispositifs de DP de type socioconstructiviste, telle la CA, entraîne des retombées sur ces autres dimensions. En effet, la réflexivité aide l'enseignant à approfondir la vision de soi et de ses valeurs éducatives, accroît les habiletés collaboratives et développe les habiletés de recherche (Dionne, 2003). Ceci est particulièrement vrai dans le cadre de groupes de recherche participative, un autre type de dispositif de DP socioconstructiviste, où les enseignants peuvent expérimenter un modèle, le modifier et le personnaliser pour se l'approprier et en accroître l'efficacité (Cormier, Pruneau, Rivard et Blain, 2004).

Dans le domaine de la pédagogie ou de l'enseignement en général, il existe une multitude de recherches qui témoignent du DP des enseignants dans une CA, ou encore dans une communauté de pratique (Cochran-Smith et Lytle, 1999; Lave et Wenger, 1991; Schussler, 2003). Bien que la littérature dans le domaine du DP en sciences soit encore relativement modeste par rapport à l'implantation de ce type de dispositifs, quelques auteurs se sont penchés sur la façon d'engager les praticiens dans la voie d'une transformation durable des pratiques pédagogiques (Erickson, 1991; Palincsar, Magnusson, Marano, Ford et Brown, 1998; Yore et Treagust, 2006). Ces auteurs suggèrent de créer des communautés d'apprentissage, des communautés de pratique ou des groupes de recherche pour aider les enseignants à ajuster leurs pratiques pédagogiques en vue d'améliorer l'apprentissage des élèves, ou encore de s'approprier des réformes. Ces dispositifs tiennent compte de l'importance de favoriser l'évolution chez l'enseignant de son rapport à l'enseignement et à l'apprentissage des sciences, ainsi que de la conception qu'il s'en fait. Dans ce sens, Yore et Treagust (2006) proposent la création de groupes de recherche ayant pour but de développer la littératie 
scientifique chez les élèves, en y intégrant un volet visant spécifiquement la maîtrise de la langue. Cette démarche tient compte des avancées en sciences cognitives, en sciences du langage et en littératies, et propose aux chercheurs et praticiens de revoir leurs diverses perspectives afin de développer un cadre d'apprentissage en sciences (Klein, 2006) au sein d'une communauté d'apprentissage didactique. Ces auteurs insistent sur l'importance de sensibiliser les enseignants à l'aspect essentiel du langage scientifique et de la démarche d'investigation dans l'enseignement des sciences.

Une étude de Palincsar, Magnusson, Marano, Ford et Brown (1998) démontre qu'une communauté de pratique peut contribuer au rehaussement de la littératie scientifique à l'école élémentaire. Le travail du groupe porte sur l'application du modèle d'investigation guidée en sciences pour le développement des littératies multiples (IGdML). Le modèle IGdLM (guided inquiry sustaining multiple litteracies GISML) vise l'intégration de changements durables dans les pratiques des enseignants. Ce modèle synergique offre une structure conceptuelle et méthodologique pour le développement d'une CA engagée dans un processus d'enrichissement des pratiques pédagogiques en sciences et en technologie (Magnusson et Palincsar, 1995; Palincsar, Magnusson, Marano, Ford et Brown, 1998) laquelle comporte trois concepts importants. Le premier, le concept d'investigation, insiste sur le fait que cette démarche de recherche est fondamentale à l'apprentissage, peu importe le domaine de connaissances. L'approche par investigation (inquiry based-learning) semble être celle qui intègre le mieux les développements récents en sciences cognitives (Palincsar, Magnusson, Marano, Ford et Brown, 1998). Le deuxième concept, celui de littératies multiples, réfère à une investigation qui prend tout son sens lorsqu'elle traverse les frontières des disciplines et, qu'en retour, les outils provenant des diverses disciplines sont mis à profit pour l'investigation. À ces deux concepts s'ajoute un troisième, celui de guidance par l'enseignant. Le modèle présuppose que le maître jouera un rôle déterminant pour aménager un environnement favorable à l'apprentissage scientifique, en mettant l'emphase sur la démarche d'investigation.

Ces deux dispositifs, celui de Yore et Treagust (2006) et celui de Palincsar et collaborateurs (1998), appliquent le modèle de l'investigation guidée en sciences au développement des littératies multiples. Dans ce contexte, une exploration des définitions et des liens qui existent entre les concepts de littératie, de littératies multiples et de littératie scientifique s'avère judicieuse avant d'aborder les stratégies et modèles pour enrichir ces littératies.

\section{Incursion sémantique autour des concepts de littératie, de littératies multiples et de littératie scientifique}

Le terme littératie renvoie à une variété de définitions parfois rattachées à des réalités fort différentes. Selon Masny (2001), bien que plusieurs considèrent ce terme comme un anglicisme utilisé en lieu et place d'alphabétisation, le concept de littératie possède en français une acception différente. Pour certains, il s'agit des capacités ou des compétences qui permettent aux individus de prendre part aux changements sociaux (Bryan, Reveles et Kelly, 2005; Roth, 2004b). Pour d'autres, il s'agit de l'acquisition de divers types de savoirs qui permet de raffiner la pensée critique chez l'individu (Klein, 1999). Une majorité de définitions fait référence à des idéologies utilitaires ou sociales. Ces définitions qualifient la littératie de fonctionnelle, ou de type humanitaire, ou de développement (Vanhulle et Schillings, 2003). La littératie désigne parfois un continuum de compétences de lecture, d'écriture ou de calcul permettant à l'individu de s'adapter à diverses situations de vie. Ces compétences vont de la maîtrise du code à la capacité d'interpréter des textes plus ou moins chargés d'implicite et faisant appel à des connaissances préalables. Les 
compétences acquises consistant à reconnaître, analyser et utiliser la langue écrite dans son fonctionnement formel, font encore qu'une personne est considérée comme lettrée ou savante.

Masny (2001) propose une définition plus globale et utilise le concept de littératies au pluriel. Ce concept réfère aux connaissances que possèdent les individus, à leur culture générale et aux ressources intellectuelles dont ils disposent. Les littératies vont au-delà de la compréhension et de l'utilisation des écrits pour englober toute une gamme de connaissances, de compétences et d'habiletés ayant trait à la lecture, aux mathématiques et aux sciences. Utilisé dans ce sens, le concept de littératies multiples s'étend à la vie quotidienne et comprend les littératies scolaire, personnelle, communautaire et critique. Celles-ci peuvent être spécifiques à une communauté ou à un individu, tributaires de l'apprentissage scolaire, ou se constituant selon un cheminement personnel, unique à chacun. Elles rejoignent la personnalité et les expériences personnelles en fonction de l'environnement dans lequel un individu évolue. Les littératies multiples permettent non seulement à l'enfant de déchiffrer les divers langages de l'école - par exemple, les langues, les mathématiques, les sciences, la musique ou l'éducation physique - et de la société, mais aussi de faire le transfert de ses connaissances dans la réalité quotidienne (Gareau-Kubicki, 1999). La capacité de penser, négocier et agir, en d'autres mots d'avoir un sens critique face à ces divers langages, s'avère un outil indispensable pour s'affirmer, décoder le monde, en dénoncer les préjugés et les stéréotypes, et participer à la transformation vers une société plus équitable (Masny, 2002). Les littératies multiples permettent d'évoquer qu'il existe des façons différentes de parler, de lire, d'écrire et de valoriser les réalités de la vie. Les littératies multiples, vues comme construit social, peuvent être considérées comme l'occasion d'utiliser un discours multiple puisqu'elles sont multi-discursives. Ces littératies tiennent compte des implications sociales, culturelles et politiques dans l'acte de lire, d'écrire et de parler, lesquelles s'opérationnalisent dans un contexte spécifique en permettant à l'individu de prendre sa place dans la société (Masny, 2001). Elles impliquent souvent des valeurs imbriquées dans des dimensions relevant de la religion, du sexe, de la culture, de l'identité ou des idéologies, et intègrent la culture et les facettes sociopolitiques et socio-historiques d'une société et de ses institutions (Masny, 2002).

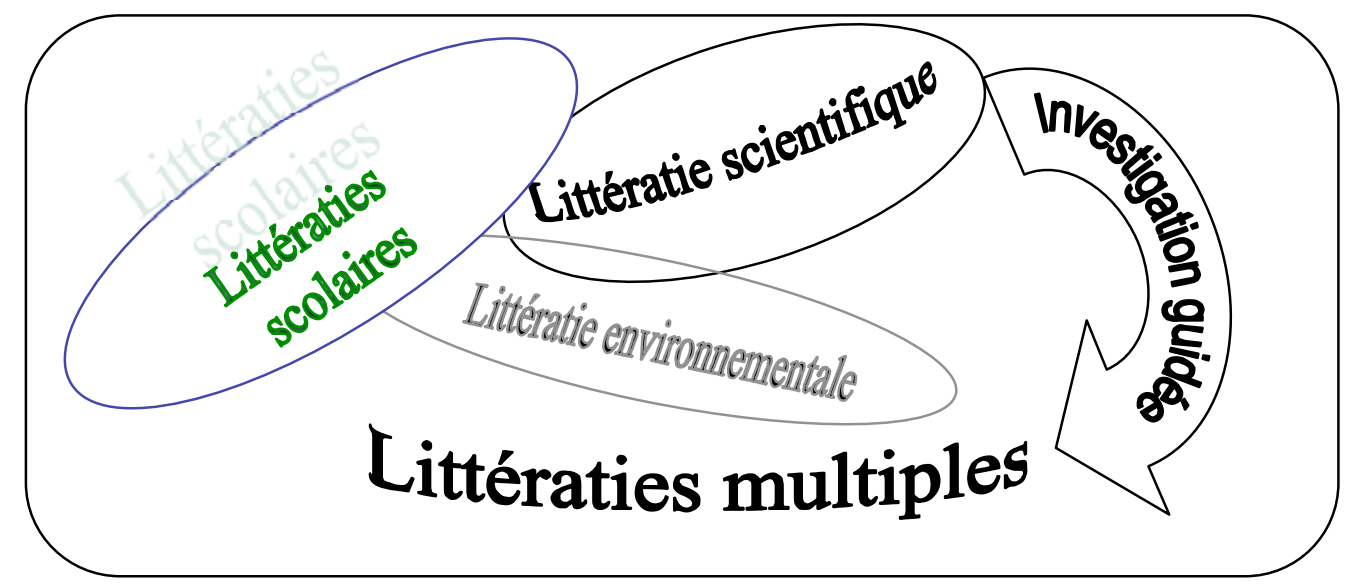

Figure 1. La relation entre la littératie scientifique et les littératies multiples 
L'apprentissage en sciences et en technologie fait partie intégrante de la culture et du développement d'un citoyen responsable et éclairé. Comme représentée dans la figure 1, la littératie scientifique ou culture scientifique, au même titre que la littératie environnementale qui lui est souvent associée, apparaît essentielle à la formation des élèves et contribue socialement au développement durable de nos sociétés (Roth, 2004a). Cette culture occupe une large place dans les littératies multiples comme construit social (Masny, 2001) et s'intègre à ces littératies surtout par la stratégie d'investigation guidée mise en place par l'enseignant en salle de classe.

La culture scientifique ou la littératie scientifique fait partie des littératies scolaires, au même titre que la numératie ou la littératie langagière. Lorsque le concept de littératie scientifique est évoqué, le domaine des sciences et de la technologie ressort plus spécifiquement. Or, selon l'OCDE (2006), la culture scientifique réfère à la capacité d'utiliser le savoir scientifique pour identifier des questions et tirer des conclusions de façon à comprendre le monde qui nous entoure et prendre des décisions pour apporter des changements dans nos activités humaines. Ce concept englobe un ensemble de connaissances individuelles et collectives dans le domaine des sciences et des technologies. La littératie scientifique sous-entend la capacité de comprendre et de raisonner d'une manière scientifique, afin de pouvoir utiliser et évaluer de façon critique les discours se rapportant aux sciences et à la technologie (Lang, Drake et Olson, 2006). Les définitions de la littératie scientifique sont multiples et vont de la référence à un modèle holistique pour améliorer l'apprentissage des sciences (Cormier, Pruneau, Rivard et Blain, 2004), aux compétences de recherche et d'utilisation du langage dans le domaine des sciences (Yore et Treagust, 2006).

Alors que la littératie générale réfère à un ensemble de compétences langagières en vue d'atteindre des buts personnels et sociaux dans des situations données (Schillings et Vanhulle, 2003), la littératie scientifique vise, quant à elle, l'utilisation de stratégies de littératies par l'élève pour décrire et expliquer ouvertement en classe ses conceptions scientifiques et technologiques (Boxie et Maring, 2002). Elle amène l'élève à mettre à l'épreuve ses conceptions en y opposant le point de vue scientifique, par le biais d'activités d'exploration et d'investigation, ce qui crée chez lui un déséquilibre cognitif nécessaire à la construction de nouvelles conceptions (Cormier Pruneau, Rivard et Blain, 2004).

Le langage en tant qu'outil de la cognition constitue un instrument de communication et d'interaction sociale dans le domaine de l'enseignement et de l'apprentissage des sciences. II permet de donner un sens à la pensée et de formuler des idées pouvant être par la suite manipulées. C'est au moyen du langage que la plupart des apprentissages de nature conceptuelle se développent. Apprendre en sciences, c'est apprendre à parler de sciences (Lemke, 1990). L'apprenant doit être en mesure d'observer et décrire les objets d'étude, d'expliquer les phénomènes observés, de concevoir et décrire la procédure d'une expérience, de formuler une conclusion et d'en faire une synthèse (Laplante, 2000). En ce sens, l'enseignement des sciences et de la technologie convie l'individu « à lire le monde » en lui offrant les stratégies de littératies multiples (Masny, 2002). Un tel enseignement possède une portée interdisciplinaire, en incitant l'élève à recourir à la dimension proprement langagière pour arriver à une compréhension conceptuelle de nature scientifique. L'élève s'assure de comprendre les concepts et sous-concepts scientifiques impliqués et d'utiliser le vocabulaire, les structures syntaxiques et les caractéristiques discursives propres à ce domaine (Laplante, 2000). La 
maîtrise des concepts scientifiques est étroitement liée aux habiletés de lire et d'écrire, qui font partie des compétences en littératie générale.

\section{Le modèle IGdML : une voie pour guider l'enseignant dans son développement professionnel en sciences et en technologie}

Un rapport produit pour le compte de l'Académie des sciences par le National Research Council (1996) aux États-Unis précise que, pour enrichir la littératie scientifique chez les élèves, les pratiques pédagogiques doivent mettre l'emphase sur trois aspects essentiels. Le premier concerne la compréhension des concepts scientifiques et le développement d'habiletés d'investigation chez l'élève. Le deuxième consiste à rendre l'apprentissage englobant et contextualisé, en aidant l'élève à approfondir les contenus de sciences et en tenant compte des perspectives personnelles, sociales et historiques entourant la nature de la science. Le troisième aspect insiste sur l'apprentissage approfondi d'un nombre plus restreint de concepts fondamentaux en sciences.

Le modèle IGdML propose à l'enseignant des stratégies pour améliorer ses pratiques en salle de classe, de façon à favoriser le changement conceptuel et l'acquisition d'habiletés scientifiques chez l'élève (Palincsar, Magnusson, Marano, Ford et Brown, 1998). De plus, en misant sur l'investigation, ce modèle nécessite de passer plus de temps à l'approfondissement de certains contenus. Les enseignants en profitent eux aussi pour approfondir les contenus et les approches. La plupart des enseignants conçoivent le langage scientifique comme étant essentiellement transparent et capable de représenter les connaissances scientifiques de façon claire et sans ambiguïté, bien que ce ne soit pas le cas. En recourant au modèle IGdML, les enseignants se rendent compte des contraintes liées au langage scientifique et surtout, à son interprétation.

De nombreuses stratégies pédagogiques peuvent être intégrées au modèle IGdML. Les propos qui suivent, ainsi que le tableau 1, en présentent quelques-unes, qui sont particulièrement adaptées au milieu minoritaire francophone. Concernant le changement conceptuel et le développement d'habiletés critiques chez l'élève, une stratégie proposée consiste à utiliser les informations provenant de l'actualité (les journaux par exemple) et à inviter l'élève à établir la distinction entre celles basées sur l'évidence scientifique, celles basées sur l'évidence non scientifique et celles basées sur l'opinion. Une autre stratégie consiste à présenter à l'élève quelques points de vue scientifiques conflictuels et à l'encourager à exprimer ses propres opinions et à porter un jugement critique (Driver, Newton et Osborne, 2000; Gayford, 2002). Ces stratégies pédagogiques visent notamment à amener l'élève à éprouver une insatisfaction à l'égard de sa conception initiale. Puis, l'enseignant lui fournit une occasion de comprendre la nouvelle conception en la trouvant plausible. Enfin, l'élève est encouragé à accepter la nouvelle conception en la jugeant susceptible d'enrichir ses connaissances. Les démarches liées à l'apprentissage mettent à profit la métacognition pour aider l'élève à faire un retour réflexif sur ses idées. Pour sa part, la stratégie d'écriture expressive, qui sert d'outil de préécriture pour l'élève avant que celui-ci n'entreprenne l'écriture scientifique proprement dite, est utilisée pour l'apprentissage en sciences en raison de sa nature constructiviste et réflexive. Celle de la lecture scientifique $\mathrm{a}$, quant à elle, pour but de former des lecteurs critiques, capables d'évaluer la validité de textes écrits en sciences (Cormier, Pruneau, Rivard, et Blain, 2004; Knain, 2006). Ces stratégies proposées en classe visent le développement d'habiletés de lecture et d'écriture en sciences, par l'établissement d'un contexte interactif permettant 
aux élèves d'expérimenter collectivement ces méthodes (Yore et Treagust, 2006). Quant à la stratégie d'apprentissage expérientiel, telle une sortie en milieu naturel, elle met en branle un processus permettant aux participants de façonner leurs conceptions par l'intermédiaire de transactions affectives et cognitives avec leurs milieux biophysique et social.

Toutes les stratégies pédagogiques illustrées qui favorisent le changement conceptuel sont basées sur les interactions sociales. Elles s'inspirent des fondements théoriques du rôle de la langue en tant qu'outil d'apprentissage (Cormier, Pruneau, Rivard et Blain, 2004). Les périodes de discussion et d'échanges au cours desquelles les interactions sociales créent une dissonance cognitive et une argumentation entre les élèves sont à favoriser. La discussion en petits groupes constitue une façon d'étayer le développement du langage, tout en favorisant simultanément le développement conceptuel en sciences. En discutant avec ses pairs, l'élève prend conscience de l'existence de conceptions différentes des siennes et il développe ses habiletés collaboratives. Dans ces stratégies, la langue occupe une place primordiale et s'intègre de façon constitutive à l'apprentissage des sciences et au changement conceptuel. C'est ce qu'on entend par faire des sciences.

Tableau 1. Stratégies pédagogiques utilisées par l'enseignant pour renforcer les liens entre sciences et littératies

\begin{tabular}{|c|c|}
\hline Stratégies pédagogiques utilisées par l'enseignant & Habiletés développées chez l'élève \\
\hline $\begin{array}{l}\text { - Utilisation de documents d'actualité ou apport } \\
\text { de points de vue scientifiques conflictuels }\end{array}$ & $\begin{array}{l}\text { Développement d'habiletés critiques, } \\
\text { changement conceptuel, métacognition, } \\
\text { habiletés collaboratives }\end{array}$ \\
\hline - Écriture expressive en contexte interactif & $\begin{array}{l}\text { Développement d'habiletés d'écriture, } \\
\text { changement conceptuel, habiletés } \\
\text { collaboratives }\end{array}$ \\
\hline - Lecture scientifique en contexte interactif & $\begin{array}{l}\text { Développement d'habiletés de lecture, } \\
\text { changement conceptuel, habiletés } \\
\text { collaboratives }\end{array}$ \\
\hline $\begin{array}{l}\text { - Apprentissage expérientiel (p. ex. sortie en } \\
\text { milieu naturel) }\end{array}$ & $\begin{array}{l}\text { - Changement conceptuel, habiletés pour } \\
\text { appréhender le milieu naturel, habiletés } \\
\text { collaboratives }\end{array}$ \\
\hline $\begin{array}{l}\text { - Discussions en petits groupes ou discussions } \\
\text { en plénière }\end{array}$ & $\begin{array}{l}\text { Développement d'habiletés d'argumentation, } \\
\text { d'habiletés critiques et changement conceptuel, } \\
\text { habiletés collaboratives }\end{array}$ \\
\hline - $\quad$ Stratégie d'investigation guidée & $\begin{array}{l}\text { - Habiletés d'investigation, identifier une } \\
\text { question, la tester, tirer des conclusions, } \\
\text { communiquer des conclusions valides et } \\
\text { démontrer sa compréhension des concepts } \\
\text { scientifiques, habiletés collaboratives, } \\
\text { changement conceptuel. }\end{array}$ \\
\hline
\end{tabular}

Ce cadre conceptuel des littératies multiples peut être mis à profit par l'enseignant pour améliorer la motivation et l'engagement des élèves. Les stratégies éducatives qui visent le développement du langage, la connaissance du discours, les connaissances en sciences et l'acquisition des habiletés scientifiques comme la démarche d'investigation (Boxie et Maring, 2002) suscitent l'intérêt des élèves. L'accent est mis sur le processus de recherche où l'élève doit préciser une question, la tester, tirer des conclusions, communiquer des conclusions valides et démontrer sa compréhension des concepts scientifiques. L'enseignant mise davantage sur l'approfondissement 
conceptuel et la démarche d'investigation pour le développement des littératies multiples, plutôt que sur la nécessité de couvrir le plus grand nombre de contenus possibles. En réalité, toutes les stratégies qui s'offrent à l'enseignant pour développer les littératies multiples, dont la littératie scientifique, peuvent s'intégrer au modèle de l'investigation guidée. L'enseignant suggère à l'élève les meilleures stratégies pour développer ses compétences à traiter l'information dans une société en mutation constante (Gayford, 2002). Ces stratégies se trouvent réunies dans le rapprochement qu'il est possible de faire entre sciences et littératies. En effet, l'élève apprend à «parler de sciences » en parlant et en argumentant au sujet des sciences. II apprend à « écrire en sciences », en écrivant sur des thèmes scientifiques pour apprendre les sciences. II apprend à «lire les sciences », en lisant des textes scientifiques pour apprendre de nouveaux concepts et explications en sciences (Laplante, 2000).

Ces stratégies ainsi que le modèle IGdML apparaissent pertinents et faciles à utiliser à la lecture de ce texte. Mais qu'en est-il de la réalité des enseignants sur le terrain? Ont-ils la possibilité d'implanter ces stratégies? Selon toute vraisemblance, les enseignants de l'élémentaire n'auraient pas la préparation adéquate et les connaissances scientifiques préalables pour intégrer de façon satisfaisante le modèle de l'investigation guidée à leur enseignement (Loucks-Horsley, Hewson, Love et Stiles, 1998). Les enseignants ont besoin de se développer professionnellement dans des dispositifs qui leur conviennent afin de rendre la science accessible, signifiante et pertinente pour leurs élèves (Lee, Hart, Cuevas et Enders, 2004). En ce sens, le modèle IGdML et toutes les stratégies présentées auparavant auraient avantage à être mises en place dans une CA didactique en sciences et en technologie, en offrant aux enseignants la possibilité de les intégrer dans la poursuite d'un projet individuel ou collectif, mais discuté collectivement. Les enseignants ont évidemment à faire un choix concernant les contenus scientifiques à aborder. II serait donc intéressant de suivre l'évolution et l'appropriation de ce modèle et de ces stratégies de littératies au cours d'une recherche empirique menée au sein d'une CA didactique en sciences et en technologie formée d'enseignants à l'élémentaire. La CA fournirait l'occasion aux enseignants de développer leurs habiletés collaboratives et d'expérimenter eux-mêmes le modèle de l'investigation guidée en sciences dans un contexte minoritaire francophone. Ils pourraient également expérimenter les stratégies de littératies discutées précédemment. Cette expérimentation de même que l'émergence d'une vision collective partagée sont à la base de l'ajustement des pratiques pédagogiques, principalement en sciences et en technologie. Ce dispositif de développement professionnel exige d'être soigneusement planifié et accompagné pour mener à une transformation durable des pratiques pédagogiques chez les enseignants (Cochran-Smith et Lytle, 1999; Palincsar, Magnusson, Marano, Ford et Brown, 1998).

\section{Conclusion}

La problématique de l'enseignement des sciences et de la technologie dans les écoles élémentaires francophones de l'Ontario va au-delà du cadre strictement curriculaire et scolaire, puisqu'elle comprend le développement professionnel des enseignants. La formation des enseignants en sciences est intimement liée à la qualité de l'enseignement dispensée (Rhoton et Stiles, 2002). Le développement professionnel des enseignants en sciences et en technologie constitue un moyen incontournable pour améliorer le niveau de littératie scientifique chez les élèves (Lee, Hart, Cuevas et Enders, 2004). L'enseignement et l'apprentissage des sciences tels qu'ils se pratiquent encore fréquemment selon un modèle traditionnel, ne donnent pas toujours le rendement escompté, comme en témoignent 
les résultats en sciences des élèves francophones en Ontario aux examens nationaux (CMEC, 2004). En ce sens, le niveau de littératie scientifique des élèves a besoin d'être rehaussé (Lee, Hart, Cuevas et Enders, 2004). L'apprentissage des sciences pourrait s'orienter davantage vers l'acquisition des compétences en littératies (processus, habiletés, savoirs être, savoirs faire, etc.) et du sens critique nécessaires aux élèves pour agir comme acteurs éclairés dans la société de demain. En fournissant les modes de pensée pour construire ce monde de demain et des réponses pour réagir aux défis de notre époque, un enseignement renouvelé pourrait provoquer un intérêt grandissant des élèves envers les sciences (Roth, 2004).

Parmi les enjeux évoqués dans cet article, il ressort le besoin de mieux contextualiser l'apprentissage des sciences et de la technologie à l'élémentaire, de mettre l'emphase sur les processus scientifiques et moins sur la somme des contenus. La formation des enseignants est ciblée comme principal moyen d'enrichir la littératie scientifique, en y introduisant des modèles de formation ou de développement professionnel porteurs pour l'intégration de pratiques pédagogiques ajustées. La CA didactique qui intègre le modèle d'investigation guidée pour le développement des littératies multiples (IGdLM) apparaît comme un dispositif qui mérite l'attention de la communauté éducative qui s'intéresse à l'éducation scientifique. Ce dispositif offre aux enseignants la possibilité de perfectionner leurs compétences en sciences et leurs compétences en didactique des sciences dans un processus d'apprentissage collectif. Ce dispositif a prouvé son efficacité dans des contextes de développement pédagogique et professionnel plus ouverts, axés sur la résolution de problèmes pédagogiques en général. Des études sont nécessaires pour mieux comprendre la particularité de ce type de dispositif dans le contexte spécifique d'un DP en enseignement des sciences, et sur la pertinence des modèles et stratégies avec lesquels les enseignants pourront travailler à ajuster leurs pratiques. Ces modèles et stratégies offrent l'avantage d'intégrer les littératies multiples (Boxie et Maring, 2002) pour orienter le changement conceptuel des élèves, contextualiser l'apprentissage des sciences, développer des compétences méthodologiques en réduisant l'accent sur les contenus, et miser sur la qualité du langage utilisé et promu en salle de classe. Il y a donc lieu d'approfondir nos connaissances concernant l'influence majeure du langage sur la pédagogie en salle de classe et sur l'apprentissage en sciences et en technologie (Lemke, 1990). Des études visant l'implantation de modèles pour guider l'innovation en matière de discussion en salle de classe ou enrichissant le développement des pratiques des enseignants dans un groupe de DP sont à envisager. Certains auteurs suggèrent qu'en proposant l'utilisation plus grande de la discussion et de la délibération en sciences dans un groupe de développement professionnel, les enseignants seront plus enclins à rehausser la qualité langagière en sciences dans leur classe (Simon, Erduran et Osborne, 2006). Ces pistes de recherche méritent d'être mieux documentées dans le contexte des écoles francophones, en particulier dans la province de l'Ontario.

\section{Références}

Abd-El-Khalick, F. et Lederman, F.G. (2000). Improving science teachers'conceptions of nature of science : A critical review of the literature. International Journal of Science Education, 22(7), 665-701.

Association des Enseignantes et des Enseignants Franco-Ontariens (AEFO) (2006). Ensemble pour la réussite! La CAP pour maintenir le cap! Projet pour l'implantation de la communauté d'apprentissage professionnelle. Document téléaccessible à l'adresse URL : http://www.aefo.on.ca/boite/publications/rapports.aspx?SID=3435E069-22F8-4C1A-B3B3-28F02C8C390B

Boxie, P. et Maring, G.H. (2002). Using web-based activities to enhance writing in science : The dynamic earth project. The Teacher Educator, 38(2), 99-111. 
Bryan, B.A., Reveles, J.M. et Kelly, G.J. (2005). Scientific literacy and discursive identity : A theoretical framework for understanding science learning. Science Education, 89(5), 779-802.

Cochran-Smith, M. et Lytle, S.L. (1999). Relationships of knowledge and practice : Teacher learning in communities. Review of Research in Education, 24, 249-305.

Cormier, M., Pruneau, D., Rivard, L. et Blain, S. (2004). Un modèle pédagogique pour améliorer l'apprentissage des sciences en milieu linguistique minoritaire. Francophonies d'Amérique, 18(1), 21-35.

Conseil des Ministres de l'Éducation du Canada (CMEC) (2004). Résultats pancanadiens des élèves francophones en milieu minoritaire au Programme d'indicateurs du rendement scolaire (PIRS). Document téléaccessible à l'adresse URL : http://www.cmec.ca/stats/publications.fr.stm

Couture, C. (2005). Repenser l'apprentissage et l'enseignement des sciences à l'école primaire. Une coconstruction entre chercheurs et praticiens. Revue des sciences de l'éducation, 31(2) 317-333.

Dionne, L. (2003). La collaboration entre collègues comme mode de développement professionnel chez l'enseignant. Thèse de doctorat inédite. Université du Québec à Montréal.

Driver, R., Newton, P. et Osborne, J. (2000). Establishing the norms of scientific argumentation in classrooms. Science Education, 84(3), 287-312.

Dufour, R. et Eaker, R. (2004). Communautés d'apprentissage professionnelles. Bloomington, Ind. : National Educational Service.

Erickson, G.L. (1991). Collaborative inquiry and the professional development of science teachers. The Journal of Educational Thought, 25(3), 228-245.

Fédération Canadienne des Enseignantes et des Enseignants (FCE) (2004). Le personnel enseignant face aux défis de l'enseignement en milieu minoritaire francophone. Rapport final de la recherche, septembre 2004. Document téléaccessible à l'adresse URL : www.circem.uottawa.ca/pdf/RevueDocumentaire.pdf

Fullan, M.G., Bennett, B. et Rolheiser-Bennett, C. (1990). Linking classroom and school improvement. Educational Leadership, 47 (8), 13-19.

Gareau-Kubicki, S. (1999). Les littératies multiples et le Programme d'éducation de maternelle. Communication présentée dans le cadre du colloque "Les recherches en littératies pour les années de formation", 67e congrès de l'ACFAS, Ottawa, ON, 10-14 mai 1999.

Gayford, C. G. (2002). Environmental Literacy : Towards a shared understanding for science teachers. Research in Science \& Technological Education, 20(1), 99-110.

Herry, Y. (2000). Enseignement et apprentissage des sciences : résultats de la troisième enquête internationale. Revue des sciences de l'éducation, 26(2), 347-366.

Klein, P.D. (1999). Learning science through writing : The role of rhetorical structures. Alberta Journal of Educational Research, 45(2), 132-153.

Klein, P.D. (2006). The Challenges of Scientific Literacy: From the viewpoint of second generation cognitive science. International Journal of Science Education, 28(2-3), 143-178.

Knain, E. (2006). Achieving science literacy through transformation of multimodal textual resources. Science Education, 90(4), 656-659.

Lang, M., Drake, S. et Olson, J. (2006). Discourse and the new didactics of scientific literacy. Journal of Curriculum Studies, 38(2), 177-188.

Laplante, B. (2000). Apprendre en sciences, c'est apprendre à " parler sciences " : des élèves de l'immersion nous parlent des réactions chimiques. Canadian Modern Language Review, 57(2), 245-271.

Larochelle, M. et Bednarz, N. (1994). À propos du constructivisme et de l'éducation. Revue des sciences de l'éducation, 20(1), 5-19.

Lave, J. et E. Wenger (1991). Situated Learning : Legitimate Peripheral Participation, New York, NY : Cambridge University Press.

Lee, O., Hart, J.E., Cuevas, P. et Enders, C. (2004). Professional development in inquiry-based science for elementary teachers of diverse student groups. Journal of Research in Science Teaching, 41(10), 1021-1043.

Lemke, J.L. (1990). Talking Science : Language, learning, and values. Norwood, NJ: Ablex Publishing Corporation.

Loucks-Horsley, S., Hewson, P.W., Love, N. et Stiles, K.E. (1998). Designing professional development for teachers of science and mathematics. Thousand Oaks, CA: Corwin Press.

Magnusson, S. et Palincsar, A.S.(1995). The learning environment as a site of science education reform. Theory into Practice, 34(1), 43-50.

Masny, D. (2002). Les littératies : un tournant dans la pensée et une façon d'être. In R. Allard (dir.), Actes du colloque pancanadien sur la recherche en éducation en milieu francophone minoritaire. Document téléacessible à l'adresse URL: http://www.acelf.ca/liens/crde/articles/14-masny.html 
Masny, D. (2001). Pour une pédagogie axée sur les littératies. In D. Masny (dir.), La culture de l'écrit : les défis à l'école et au foyer (15-26). Montréal : Les Éditions Logiques. Document téléacessible à l'adresse URL : http:/laix1.uottawa.ca/ dmasny/french/writings/index.html

Morais, A., Neves, I. et Afonso, M. (2005). Teacher training processes and teachers' competence : A sociological study in the primary school. Teaching and Teacher Education : An International Journal of Research and Studies, 21(4), 415-437.

National Research Council (NRC) (1996). National Science Education Standards. Washington, DC : National Academic Press.

OCDE (2006). Assessing scientific, reading and mathematic literacy: A framework for PISA (Programme for International Student Assessment) 2006. OCDE Publishing. Document téléaccessible à l'adresse URL : http://213.253.134.23/oecd/display.asp?K=5L9PX1W9DN7J\&lang=EN\&sort=sort date\%2Fd\&sf1=kwords\&st1=defini tion+scientific+literacy\&sf3=SubjectCode\&st4=not+E4+or+E5+or+P5\&sf4=SubVersionCode\&ds=definition+scientif ic+literacy\%3B+All+Subjects\%3B+\&m=1\&dc=2\&plang=en

Palincsar, A. S., Collins, K., Marano, N., Magnusson, S. (2000). Investigating the engagement and learning of students with learning disabilities in guided inquiry science teaching. Language, Speech, and Hearing Services in Schools, 31, 240-251.

Palincsar, A., Magnusson, S.J., Marano, N., Ford, D. et Brown, N. (1998). Designing a community of practice : principles and practices of the GISML community. Teaching and Teacher Education, 14(1), 5-19.

Rhoton, J. et Stiles, E. K. (2002). Exploring the Professional Development Design Process : Bringing an Abstract Framework into Practice. Science Educator, 11(1), 1-8.

Roth, W.-M. (2004). Science as Collective Praxis, Literacy, Power, and Struggle for a Better World. In W.-M. Roth et A. Calabrese Barton (dir.), Rethinking scientific literacy (p. 1-20). New York, NY : Routledge Falmer.

Roth, W.-M. (2004a). Scientific literacy as emergent feature of collective praxis. In W.-M. Roth et A. Calabrese Barton (dir.), Rethinking scientific literacy (p. 21-48). New York, NY : Routledge Falmer.

Roth, W.-M. (2004b). Scientific literacy, hegemony, and struggle. In W.-M. Roth et A. Calabrese Barton (dir.), Rethinking scientific literacy (p. 49-76). New York, NY : Routledge Falmer.

Schussler, D.L. (2003). Schools as learning communities : Unpacking the concept. Journal of School Leadership, 13, 498-528.

Savoie-Zajc, L. et Descamps-Bednarz, N. (2007). Action research and collaborative research : Their specific contributions to professional development. Educational Action Research, 15(4), 577-596.

Savoie-Zajc, L. et Dionne, L. (2006). Towards a definition of learning community in schools. Communication présentée à la Conférence Internationale ECER, European Educational Research Association (EERA), Genève, 13 septembre 2006.

Seidel Horn, I. (2003). Learning on the job : A situated account of teacher learning in high school mathematics departments. Cognition and Instruction, 23(2), 1-18.

Simon, S., Erduran, S. et Osborne, J. (2006). Learning to teach argumentation : Research and development in the science classroom. International Journal of Science Education, 28(2-3), 235-260.

Sparks, D. (1999). Real-life-view. Journal of Staff Development, 20(4), 53-57.

Tobin, K., Tippins, D.J., et Gallard, A.J. (1994). Research on instructional strategies for teaching science. In D.L. Gabel (dir.), Handbook of Research on Science Teaching and Learning (p.3-44). New York, NY : Macmillan.

Vanhulle, S. et Schillings, A. (2003). La « littératie » métaphores idéologiques ou concept didactique. Service de pédagogie expérimentale, Université de Liège. Document téléacessible à l'adresse URL : http://www.enseignement.be/@librairie/documents/ressources/A005/rapfin/biblio.asp

Wasley, P.A., Donmoyer, R. et Maxwell, L. (1995). Navigating change in high school science and mathematics : Lessons teachers taught us. Theory into Practice, 34(1), 51-59.

Wells, G. (1993). Working with a teacher in the zone of proximal development : Action research on the learning and teaching of science. Journal of the Society for Accelerative Learning and Teaching, 18 (1/2), 127-222.

Yore, L. et Treagust, D. (2006). Current Realities and Future Possibilities: Language and science literacy. Empowering research and informing instruction. International Journal of Science Education, 28(2-3), 291-314. 